\title{
On a conjecture of E. Thomas concerning parametrized Thue equations
}

\author{
by \\ Clemens Heuberger (Graz)
}

1. Introduction. For an irreducible form $F \in \mathbb{Z}[X, Y]$ of degree at least 3 and a nonzero integer $m$, the Diophantine equation

$$
F(X, Y)=m
$$

is called a Thue equation in honour of A. Thue, who proved in 1909 [19] that the number of its solutions in integers is finite. Thue's result is not effective, but in 1968, A. Baker [1] gave an upper bound for the solutions using his lower bounds for linear forms in logarithms of algebraic numbers. Since then, algorithms for the solution of single Thue equations have been developed (see Pethő and Schulenberg [14], Tzanakis and de Weger [20], and Bilu and Hanrot [4]).

In 1990, E. Thomas [17] considered for the first time a parametrized family of cubic Thue equations of positive discriminant. In the last decade, several such families of degrees 3 to 6 have been investigated (see [8] for further references). In all these families, there were only two types of solutions: Firstly, there are polynomial solutions $X(a), Y(a) \in \mathbb{Z}[a]$ which satisfy $F(X, Y)= \pm 1$ in $\mathbb{Z}[a]$, and secondly, there may be some further solutions for special (small) values of the parameter.

The family of Thue equations

$$
a X^{n}-b Y^{n}= \pm 1
$$

has been considered by several authors. Bennett [3] recently showed that for $a b \neq 0$ and $n \geq 3$ this equation has at most one solution in positive integers $(x, y)$.

A further step is the investigation of classes of parametrized families of arbitrary degree such as

2000 Mathematics Subject Classification: Primary 11D59.

This work was supported by Austrian National Bank project nr. 7088 and the Austrian-Hungarian Science Cooperation project. 


$$
F_{a}(X, Y):=\prod_{i=1}^{n}\left(X-p_{i}(a) Y\right)-Y^{n}= \pm 1, \quad a \in \mathbb{N},
$$

where $p_{1}, \ldots, p_{n} \in \mathbb{Z}[a]$ are polynomials, which have been called split families by E. Thomas [18]. For $i=1, \ldots, n$ it can be easily seen that $(X, Y) \in$ $\left\{ \pm\left(p_{i}, 1\right),( \pm 1,0)\right\}$ are polynomial solutions. Thomas conjectured that if

$$
p_{1}=0, \quad 0<\operatorname{deg} p_{2}<\ldots<\operatorname{deg} p_{n}
$$

and the polynomials are monic, there are no further solutions for sufficiently large values of the parameter $a$. In [18] he proved this conjecture for $n=3$ under some technical hypothesis.

Halter-Koch, Lettl, Pethő and Tichy [6] considered (1) for $p_{1}=0, p_{2}=$ $d_{2}, \ldots, p_{n-1}=d_{n-1}$ and $p_{n}=a$, where $d_{2}, \ldots, d_{n-1}$ are distinct fixed integers. They found all solutions for sufficiently large values of $a$ assuming a conjecture of Lang and Waldschmidt [10] — which is a very sharp bound for linear forms in logarithms of algebraic numbers - provided that the corresponding number field is primitive (i.e. $\mathbb{Q}$ is the only proper subfield), which is the case for almost all choices (in the sense of thin sets) of the parameters.

The first unconditional result on split families of arbitrary degree has been given in [7], where (1) has been considered for $p_{1}=-a, p_{2}=d_{2}, \ldots$ $\ldots, p_{n-1}=d_{n-1}$ and $p_{n}=a$, where $d_{2}, \ldots, d_{n-1}$ are distinct fixed integers. If $\sum_{i} d_{i} \neq 0$ or $\prod_{i} d_{i} \neq 0$, then the only solutions are polynomial solutions with $|Y| \leq 1$.

In [9], Heuberger and Tichy considered a multivariate version of (1): Let $p_{i} \in \mathbb{Z}\left[a_{1}, \ldots, a_{r}\right]$ for some $r \in \mathbb{N}$. Assume

$$
\begin{gathered}
\operatorname{deg} p_{1}<\ldots<\operatorname{deg} p_{n-2}<\operatorname{deg} p_{n-1}=\operatorname{deg} p_{n}, \\
\operatorname{LH}\left(p_{n}\right)=\operatorname{LH}\left(p_{n-1}\right), \quad \text { but } p_{n} \neq p_{n-1},
\end{gathered}
$$

where $\operatorname{LH}(p)$ denotes the homogeneous part of maximal degree of a polynomial $p$, and suppose that the polynomials $p_{i}$ satisfy suitable growth conditions. Then there is a constant $t_{0}$ such that for all $a_{1}, \ldots, a_{r}$ satisfying $t_{0} \leq \min _{k} a_{k}$ and $\max _{k} a_{k} \leq\left(\min _{k} a_{k}\right)^{\tau}$ the Diophantine equation (1) has only polynomial solutions with $|Y| \leq 1$, where $\tau>1$ is an explicitly given constant (depending on the degrees of the polynomials only).

These two results depend heavily on the symmetric nature of the polynomials $p_{i}$. In this paper, we shall prove Thomas' conjecture for a very large class of polynomials $p_{i}$, subject only to certain technical conditions on the degrees of the $p_{i}$.

After the presentation of the results in this section we shall, in Section 2, give some comments on the technical hypothesis occurring in our Theorems 1 and 2 and we will discuss its relation to Thomas' technical hypothesis in the case $n=3$. In Sections 3 and 4 we collect and adapt standard material for the solution of parametrized Thue equations. In Section 5 we will present 
the main idea to exclude "small" solutions, which will be carried out in detail in Sections 6 and 7. In Section 8 we will exclude "large" solutions using Baker's method via application of a result of Bugeaud and Győry [5]. Finally we will prove a weaker formulation of the technical hypothesis in Section 9.

The main result of the present paper is

TheOREM 1. Let $n \in \mathbb{N}, n \geq 4$ and $p_{i} \in \mathbb{Z}[a]$ be monic polynomials for $i=1, \ldots, n$. Write $d_{i}:=\operatorname{deg} p_{i}$,

$$
e_{i}:=(i-1) d_{i}+\sum_{l=i+1}^{n} d_{l}
$$

and allow $p_{1}=0$ (set $d_{1}=-1$ in this case). Moreover, define recursively

$$
\psi_{i}:=\frac{\left(e_{2}+d_{2}\right)\left(d_{i+1}-d_{3}\right)}{e_{i+1}+d_{i+1}}+\sum_{h=3}^{i-1} \frac{d_{i+1}-d_{h+1}}{e_{i+1}+d_{i+1}} \psi_{h}, \quad 3 \leq i \leq n-1 .
$$

If $\psi_{i} \in \mathbb{N}$ for all $3 \leq i \leq n-1$, for $\left(j, j^{\prime}\right) \in\{(1,2),(2,1)\}$ define

$$
\begin{aligned}
& Q_{j}^{+}:=\left(p_{3}-p_{j}\right)^{e_{1}+d_{3}} \prod_{k=4}^{n}\left(p_{k}-p_{3}\right)^{\psi_{k-1}}, \\
& Q_{j}^{-}:=\left(p_{2}-p_{1}\right)^{e_{1}+2 d_{3}-d_{2}}\left(p_{3}-p_{j^{\prime}}\right)^{2\left(d_{3}-d_{2}\right)} \prod_{k=4}^{n}\left(p_{k}-p_{j^{\prime}}\right)^{\psi_{k-1}+d_{3}-d_{2}} .
\end{aligned}
$$

Assume

$$
d_{1}<d_{2}<\ldots<d_{n-1}<d_{n} .
$$

If there is a $3 \leq k \leq n-1$ such that $\psi_{k} \notin \mathbb{N}$ or if

$$
\operatorname{deg}\left(Q_{j}^{+}-Q_{j}^{-}\right)>\operatorname{deg} Q_{j}^{-}-e_{1}-d_{2}
$$

for $\left(j, j^{\prime}\right)=(1,2)$ and for $\left(j, j^{\prime}\right)=(2,1)$, then there is a (computable) constant $a_{0}=a_{0}\left(p_{1}, \ldots, p_{n}\right)$ depending on the coefficients of the polynomials $p_{i}$ such that for all integers $a \geq a_{0}$ the Diophantine equation

$$
F_{a}(X, Y):=\prod_{i=1}^{n}\left(X-p_{i}(a) Y\right)-Y^{n}= \pm 1
$$

has only the solutions

$$
( \pm 1,0) \quad \text { and } \pm\left(p_{i}(a), 1\right), \quad 1 \leq i \leq n .
$$

The case $n=3$ has been excluded in the formulation of Theorem 1 in order to avoid any ambiguities; it is stated explicitly in the following theorem:

Theorem 2. Let $p_{1}, p_{2}, p_{3} \in \mathbb{Z}[a]$ be monic polynomials. Write $d_{i}:=$ $\operatorname{deg} p_{i}$

$$
e_{i}:=(i-1) d_{i}+\sum_{l=i+1}^{3} d_{l}
$$


and allow $p_{1}=0$ (set $d_{1}=-1$ in this case). For $\left(j, j^{\prime}\right) \in\{(1,2),(2,1)\}$ define

$$
Q_{j}^{+}:=\left(p_{3}-p_{j}\right)^{e_{1}+d_{3}}, \quad Q_{j}^{-}:=\left(p_{2}-p_{1}\right)^{e_{1}+2 d_{3}-d_{2}}\left(p_{3}-p_{j^{\prime}}\right)^{2\left(d_{3}-d_{2}\right)} .
$$

Assume

$$
d_{1}<d_{2}<d_{3} \quad \text { and } \quad d_{2} \geq 1
$$

If

$$
\operatorname{deg}\left(Q_{j}^{+}-Q_{j}^{-}\right)>\operatorname{deg} Q_{j}^{-}-e_{1}-d_{2}
$$

for $\left(j, j^{\prime}\right)=(1,2)$ and for $\left(j, j^{\prime}\right)=(2,1)$, then there is a (computable) constant $a_{0}=a_{0}\left(p_{1}, p_{2}, p_{3}\right)$ depending on the coefficients of the polynomials $p_{i}$ such that for all integers $a \geq a_{0}$ the Diophantine equation

$$
F_{a}(X, Y):=\prod_{i=1}^{3}\left(X-p_{i}(a) Y\right)-Y^{n}= \pm 1
$$

has only the solutions

$$
( \pm 1,0) \quad \text { and } \pm\left(p_{i}(a), 1\right), \quad 1 \leq i \leq 3 .
$$

We remark that if $d_{2}=0$, i.e. $p_{1}=0, p_{2}=1$ and $\operatorname{deg} p_{3} \geq 1$, the assertion of the theorem is false since $F_{a}\left(p_{3}-3, p_{3}-2\right)=-1$. See however Lee [11], Mignotte and Tzanakis [13], and Mignotte [12], where all solutions of this family are determined.

We also give a weaker formulation of the technical hypothesis:

Corollary 3. Let $n \in \mathbb{N}, n \geq 3$ and $p_{i} \in \mathbb{Z}[a]$ be monic polynomials for $i=1, \ldots, n$. Write

$$
p_{i}(a)=a^{d_{i}}+c_{i} a^{d_{i}-1}+\text { terms of lower degree, } \quad i=2, \ldots, n,
$$

allow $p_{1}=0$ and assume (4). If $n=3$, assume (8). Let

$$
\delta_{i}:= \begin{cases}1 & \text { if } d_{i}-d_{i-1}=1 \\ 0 & \text { otherwise }\end{cases}
$$

and

If $\delta_{4}=1$ or

$$
e:=\sum_{i=2}^{n} d_{i}
$$

$$
\begin{aligned}
\left(e-d_{2}+2 d_{3}\right)\left(c_{2}-\delta_{2}\right)+\left(-e-2 d_{2}+d_{3}\right) c_{3} & +\left(d_{3}-d_{2}\right) \sum_{i=4}^{n} c_{i} \\
& \notin\left\{2 \delta_{3},-\left(e+d_{3}\right) \delta_{3}\right\},
\end{aligned}
$$

then there is a (computable) constant $a_{0}=a_{0}\left(p_{1}, \ldots, p_{n}\right)$ depending on the coefficients of the polynomials $p_{i}$ such that for all integers $a \geq a_{0}$ the Diophantine equation (6) has only the solutions (7).

If the technical hypothesis in Theorem 1 is checked for small degrees, the following corollary is obtained. 
Corollary 4. Let $n \in \mathbb{N}, n \in\{4,5\}$ and $p_{i} \in \mathbb{Z}[a]$ be monic polynomials for $i=1, \ldots, n$, where we allow $p_{1}=0$. If

$$
\begin{gathered}
\max \left(\operatorname{deg} p_{1}, 0\right)<\operatorname{deg} p_{2}<\operatorname{deg} p_{3}<\ldots<\operatorname{deg} p_{n}, \\
\max \left(\operatorname{deg} p_{1}, 0\right)+\operatorname{deg} p_{2}+\ldots+\operatorname{deg} p_{n}<15,
\end{gathered}
$$

then there is a (computable) constant $a_{0}=a_{0}\left(p_{1}, \ldots, p_{n}\right)$ depending on the coefficients of the polynomials $p_{i}$ such that for all integers $a \geq a_{0}$ the Diophantine equation

$$
F_{a}(X, Y)=\prod_{i=1}^{n}\left(X-p_{i}(a) Y\right)-Y^{n}= \pm 1
$$

has only the solutions (7).

Note that for $n \geq 6$ condition (12) cannot hold since $\sum \operatorname{deg} p_{i} \geq 0+1+\ldots$ $\ldots+(n-1)$.

2. About the technical hypothesis. Obviously, it is easy to check the technical hypothesis in Theorems 1 and 2 for a given set of polynomials $p_{i}$. Therefore we shall discuss the problem with given degrees $d_{1}, \ldots, d_{n}$ and unknown coefficients of the polynomials $p_{i}$. By the definition of $\psi_{i}$ in (3) we see that it is rather unlikely that all $\psi_{i}$ are integers, especially if $n$ is large.

If the $\psi_{i}$ are indeed all integers, (5) implies that the $\max \left(0, d_{1}\right)+\sum_{i=2}^{n} d_{i}$ unknown coefficients satisfy a system of $e_{1}+d_{2}-1$ algebraic equations. We may further assume that the coefficient of $a^{d_{n}-1}$ in $p_{n}(a)$ vanishes, since we can replace the problem involving $p_{i}(a)$ by that involving $p_{i}(a-1)$.

Hence we have $e_{1}+d_{2}-1$ equations in $e_{1}+\max \left(0, d_{1}\right)-1$ unknowns; if we exclude $\left(d_{1}, d_{2}\right)=(-\infty, 0)$ - which has also been excluded by Thomas in the statement of his conjecture - we have an overdetermined system of algebraic equations. Of course, this system is expected not to have even complex solutions and integer solutions seem to be rather unlikely.

The result of such studies for small degrees is given in Corollary 4, where we excluded again the case $\left(d_{1}, d_{2}\right)=(-\infty, 0)$.

Proof of Corollary 4. In order to prove Corollary 4 we only have to check the assumptions of Theorem 1 . For $n=5$, there are no degrees $\operatorname{deg} p_{i}$ such that (12), $\psi_{3} \in \mathbb{N}$ and $\psi_{4} \in \mathbb{N}$ hold.

For $n=4$, there are only 13 sets $\left\{d_{1}, \ldots, d_{4}\right\}$ which satisfy both (12) and $\psi_{3} \in \mathbb{N}$. For those degrees we calculate the systems of algebraic equations corresponding to (5) modulo 5 and 7 (using Pari [2]) and calculate a reduced Gröbner basis over $\mathbb{Z}_{m}(m \in\{5,7\})$ with respect to the total degree ordering of the power products (using the program Gröbner [21]). In all cases, the Gröbner basis consists for at least one of the moduli 5 and 7 of the polynomial 1 only. Therefore the systems of algebraic equations have no integer solutions, which proves (5); thus the assertion follows from Theorem 1. 
In order to compare the technical hypothesis in Theorem 2 with Thomas' technical hypothesis [18], we note that in the case considered in [18], we have $p_{1}=0$ and $\operatorname{deg} p_{2}>0$. Obviously, (9) is equivalent to

We calculate

$$
\log \frac{Q_{j}^{-}}{Q_{j}^{+}}=\log \left(1+\frac{Q_{j}^{-}-Q_{j}^{+}}{Q_{j}^{+}}\right)=\Omega\left(\frac{1}{a^{e_{1}+d_{2}-1}}\right) .
$$

$$
\begin{aligned}
\log \left(\frac{Q_{1}^{-}}{Q_{1}^{+}}\right) & =3 \log \frac{p_{2}^{d_{3}}}{p_{3}^{d_{2}}}+2\left(d_{3}-d_{2}\right) \log \left(1-\frac{p_{2}}{p_{3}}\right) \\
& =3 W(a)-2\left(d_{3}-d_{2}\right) a^{-\left(d_{3}-d_{2}\right)}\left(1+O\left(a^{-1}\right)\right)
\end{aligned}
$$

and

$$
\begin{aligned}
\log \left(\frac{Q_{2}^{-}}{Q_{2}^{+}}\right) & =3 \log \frac{p_{2}^{d_{3}}}{p_{3}^{d_{2}}}-\left(d_{2}+2 d_{3}\right) \log \left(1-\frac{p_{2}}{p_{3}}\right) \\
& =3 W(a)+\left(d_{2}+2 d_{3}\right) a^{-\left(d_{3}-d_{2}\right)}\left(1+O\left(a^{-1}\right)\right),
\end{aligned}
$$

where

$$
\begin{gathered}
1+P_{i}(a)=p_{i} / a^{d_{i}}, \quad i=2,3, \\
W(a)=\sum_{k=1}^{\infty} \frac{(-1)^{k+1}}{k}\left(d_{3} P_{2}^{k}-d_{2} P_{3}^{k}\right)=\sum_{k=1}^{\infty} \frac{w_{k}}{a^{k}}
\end{gathered}
$$

is as in $[18,(1.9)-(1.11)]$. It is easily seen that Thomas' definition of a regular family $[18,(1.12)]$ is equivalent to

$$
\log \left(\frac{Q_{j}^{-}}{Q_{j}^{+}}\right)=\Omega\left(\frac{1}{a^{d_{3}-d_{2}}}\right) .
$$

Since $d_{3}-d_{2}<e_{1}+d_{2}-1=2 d_{2}+d_{3}-1$, this implies (9), therefore Theorem 2 improves Thomas' Theorem 2.

3. Preliminaries. We first consider solutions $(x, y)$ with $|y| \leq 1$.

Lemma 5. The solutions $(x, y) \in \mathbb{Z}^{2}$ of (6) with $|y| \leq 1$ are precisely those listed in (7), if a is large enough.

Proof. The pairs $(x, y)$ listed in (7) are clearly solutions of (6).

Conversely, $y=0$ implies $x= \pm 1$. If $|y|=1$, we have $\prod\left(x-p_{i}(a) y\right)=0$, which yields the solutions listed in (7), or

$$
\prod_{i=1}^{n}\left(x-p_{i}(a) y\right)=2 u, \quad u= \pm 1 .
$$

By (4) the factors of this product are pairwise distinct for sufficiently large $a$, and consequently for $n \geq 4,(13)$ is impossible.

Assume $n=3$. Then we may have $x-p_{1}(a) y=k_{1}, x-p_{2}(a) y=k_{2}$, $x-p_{3}(a) y=k_{3}$ for $\left\{k_{1}, k_{2}, k_{3}\right\}=\{-1,1,-2 u\}$. This implies that 
$\left(p_{3}(a)-p_{2}(a)\right) y-\left(k_{2}-k_{3}\right)=0$, which has only a finite number of solutions $a$ by (4), thus (13) has no solutions for large $a$.

Consider the polynomial $f_{a}(X):=F_{a}(X, 1)$. We will need asymptotic estimates for its roots $\alpha^{(1)}, \ldots, \alpha^{(n)}$. Throughout this paper, we will use $O$-, $\Omega$ - and $\Theta$-notation for $a \rightarrow \infty$ : Let $f, g: \mathbb{R} \rightarrow \mathbb{R}$. If there are constants $C$ and $a_{0}$ such that $|f(a)| \leq g(a)$ (resp. $|f(a)| \geq g(a)$ ) for all $a \geq a_{0}$, we write $f(a)=O(g(a))$ (resp. $f(a)=\Omega(g(a)))$. If both $f(a)=O(g(a))$ and $f(a)=\Omega(g(a))$ hold, then we write $f(a)=\Theta(g(a))$. For brevity, we will write $p_{i}$ instead of $p_{i}(a)$ in many situations.

We get a statement which is similar to that of Lemma 5 of [9]:

Lemma 6. All roots of $f_{a}(X)$ are real and can be estimated as

$$
\alpha^{(i)}=p_{i}+\frac{(-1)^{n-i}}{a^{e_{i}}}+O\left(\frac{1}{a^{e_{i}+1}}\right), \quad 1 \leq i \leq n .
$$

Proof. We fix some $1 \leq i \leq n$ and set

$$
\alpha_{i, M}:=p_{i}+\frac{(-1)^{n-i}}{a^{e_{i}}}\left(1+\frac{M}{a}\right),
$$

where $M$ is a constant which will be chosen later.

Equality (2) and inequality (4) imply

$$
e_{i}=(i-1) d_{i}+\sum_{j=i+1}^{n} d_{j}=\sum_{j \neq i} \operatorname{deg}\left(p_{i}-p_{j}\right)
$$

and $e_{i} \geq 3$ for $n \geq 3$.

This yields for $j \neq i$,

$$
\alpha_{i, M}-p_{j}=a^{\operatorname{deg}\left(p_{i}-p_{j}\right)}(-1)^{\sigma_{i j}}\left(1+\frac{c_{i j}}{a}+O\left(\frac{1}{a^{2}}\right)\right),
$$

where $c_{i j} \in \mathbb{Z}$ depends on the coefficients of $p_{i}$ and $p_{j}$ and

$$
\sigma_{i j}= \begin{cases}0 & \text { if } i>j \\ 1 & \text { if } i<j\end{cases}
$$

We calculate an asymptotic expansion for $f\left(\alpha_{i, M}\right)$ using (14) and (15):

$$
\begin{aligned}
f\left(\alpha_{i, M}\right) & =\frac{(-1)^{n-i}}{a^{e_{i}}}\left(1+\frac{M}{a}\right) \prod_{j \neq i}\left(\alpha_{i, M}-p_{j}\right)-1 \\
& =\left(1+\frac{M}{a}\right) \prod_{j \neq i}\left(1+\frac{c_{i j}}{a}+O\left(\frac{1}{a^{2}}\right)\right)-1 \\
& =\frac{1}{a}\left(M+\sum_{j \neq i} c_{i j}\right)+O\left(\frac{1}{a^{2}}\right) .
\end{aligned}
$$


We can choose constants $M_{1}$ and $M_{2}$ in such a way that $M_{1}+\sum_{j \neq i} c_{i j}>0$ and $M_{2}+\sum_{j \neq i} c_{i j}<0$, which implies that $f\left(\alpha_{i, M_{1}}\right) f\left(\alpha_{i, M_{2}}\right)<0$ for sufficiently large $a$. As a result we have found a zero of $f$ between $\alpha_{i, M_{1}}$ and $\alpha_{i, M_{2}}$ if $a$ is large enough.

4. Associated number field. Since $f_{a}$ is an irreducible polynomial for sufficiently large $a$ by [7, Proposition 3] and (4), the number field $K:=\mathbb{Q}(\alpha)$ generated by one of the roots $\alpha$ of $f_{a}$ has degree $n$ over $\mathbb{Q}$.

If $(x, y) \in \mathbb{Z}^{2}$ is a solution of $(6)$, then

$$
N_{K / \mathbb{Q}}(x-\alpha y)=F(x, y)= \pm 1,
$$

hence $x-\alpha y$ is a unit in $\mathfrak{O}:=\mathbb{Z}[\alpha]$. Therefore, we will describe the structure of the unit group $\mathfrak{O}^{\times}$.

We define

$$
\eta_{i}:=\alpha-p_{i}
$$

Since $f_{a}(\alpha)=0$ we have $\prod_{i} \eta_{i}=1$, which implies that $\eta_{i}$ is a unit in $\mathfrak{O}$.

We will show that $\eta_{i}, i=1, \ldots, n-1$, are "sufficiently close" to fundamental units of $\mathfrak{O}^{\times}$. In order to estimate the index of the group generated by these units, we will need a lower bound for the regulator of the unit group. Since logarithmic terms do not matter for our purposes, we can take any regulator estimate, for instance that of Remak [16].

LEMMA 7 (Remak). Let $K$ be a totally real number field. Then the regulator $R_{K}$ satisfies

$$
R_{K}>0.001
$$

We give an asymptotic description of the $\eta_{i}^{(k)}$. To simplify notation, we define the abbreviation $l_{i}^{(k)}:=\log \left|\eta_{i}^{(k)}\right|$.

Lemma 8. Let $1 \leq i, k \leq n, m:=\min (i, k)$ and $M:=\max (i, k)$. Then

$$
l_{i}^{(k)}=\log \left(p_{M}-p_{m}\right)+O\left(\frac{1}{a^{e_{m}+d_{M}}}\right), \quad i \neq k .
$$

Moreover, there are $r_{M, m, l} \in \mathbb{Q}$ for $l=0, \ldots, e_{1}+d_{M}-1$ depending only on the coefficients of $p_{s}, s=1, \ldots, n$, such that

$$
l_{i}^{(k)}=r_{M, m, 0} \log a+\sum_{l=1}^{e_{1}+d_{M}-1} \frac{r_{M, m, l}}{a^{l}}+O\left(\frac{1}{a^{e_{1}+d_{M}}}\right) .
$$

In particular,

$$
l_{i}^{(k)}= \begin{cases}d_{M} \log a+O(1 / a) & \text { if } i \neq k \\ -e_{i} \log a+O(1 / a) & \text { if } i=k\end{cases}
$$


Proof. Assume $i \neq k$. By Lemma 6 we obtain

$$
\begin{aligned}
l_{i}^{(k)} & =\log \left|\alpha^{(k)}-p_{i}\right|=\log \left|p_{k}-p_{i}+O\left(a^{-e_{k}}\right)\right|=\log \left(p_{M}-p_{m}+O\left(a^{-e_{m}}\right)\right) \\
& =\log \left[\left(p_{M}-p_{m}\right)\left(1+O\left(a^{-e_{m}-d_{M}}\right)\right)\right]=\log \left(p_{M}-p_{m}\right)+O\left(a^{-e_{m}-d_{M}}\right) .
\end{aligned}
$$

The power series expansion of $\log (1+z)$ yields the assertions.

Let now $i=k$. By definition of $\alpha^{(i)}$ we have $l_{i}^{(i)}=-\sum_{j \neq i} l_{j}^{(i)}$, and the lemma is proved.

Lemma 9. Let $\left\{i_{1}, \ldots, i_{n-1}\right\}$ be a subset of $\{1, \ldots, n\}$ of cardinality $n-1$ and

$$
G:=\left\langle-1, \eta_{i_{1}}, \ldots, \eta_{i_{n-1}}\right\rangle \subseteq \mathfrak{O}^{\times} .
$$

Then the regulator $R_{G}$ can be estimated by

$$
R_{G}=\Theta\left(\log ^{n-1} a\right)
$$

and the index $\left[\mathfrak{O}^{\times}: G\right]$ is bounded by

$$
\left[\mathfrak{O}^{\times}: G\right]=O\left(\log ^{n-1} a\right) .
$$

Proof. Assume first $i_{1}=1, \ldots, i_{n-1}=n-1$. Then (17) follows from Lemma 8 and $[9$, Lemma 7$]$. For arbitrary $i_{1}, \ldots, i_{n-1}$, the result follows from $l_{n}^{(i)}=-\sum_{k=1}^{n-1} l_{k}^{(i)}$.

Equality (18) is a consequence of Pohst and Zassenhaus [15, p. 361], Lemma 7 , and (17):

$$
I=\left[\mathfrak{O}^{\times}: G\right]=\frac{R_{G}}{R_{\mathfrak{O}}} \leq \frac{R_{G}}{R_{\mathfrak{O}_{K}}} \leq \frac{R_{G}}{0.001}=O\left(\log ^{n-1} a\right) .
$$

5. Approximation properties of solutions. Let $(x, y) \in \mathbb{Z}^{2}$ be a solution of (6) and $\beta:=x-\alpha y$. We define the type $j$ of a solution $(x, y)$ such that

$$
\left|\beta^{(j)}\right|=\min _{i=1, \ldots, n}\left|\beta^{(i)}\right| .
$$

By $[9$, Proof of Lemma 12] we obtain

$$
\left|\frac{\beta^{(j)}}{y}\right|=O\left(\frac{1}{a^{e_{j}}}\right)
$$

hence for $i \neq j$,

$$
\left|\frac{\beta^{(i)}}{y}\right|=\left|\frac{x}{y}-\alpha^{(j)}+\alpha^{(j)}-p_{j}+p_{j}-\alpha^{(i)}\right|=\left|\eta_{j}^{(i)}\right|\left(1+O\left(\frac{1}{a^{e_{j}+d_{2}}}\right)\right),
$$

and therefore

$$
\log \left|\beta^{(i)}\right|=\log |y|+l_{j}^{(i)}+O\left(\frac{1}{a^{e_{j}+d_{2}}}\right), \quad i \neq j .
$$


The crucial part of the proof of Theorems 1 and 2 will be the following proposition, which shows that only solutions with $|y| \leq 1$ or very large $|y|$ can exist. The latter can be excluded easily by standard methods (see Section 8).

Proposition 10. Let $(x, y) \in \mathbb{Z}^{2}$ be a solution of (6) with $|y| \geq 2$. Then

$$
\log |y|=\Omega\left(\frac{a}{\log ^{n-3} a}\right) .
$$

This proposition will be proved as follows: First we give the parts of the proof which are independent of the type $j$ of the solution, whereas the estimates which depend on the type will be provided in the following sections.

Since $\beta$ is a unit by (16), Lemma 9 yields

$$
\beta^{I}= \pm \eta_{i_{1}}^{u_{i_{1}}} \ldots \eta_{i_{n-1}}^{u_{i_{n-1}}}
$$

where $\left\{i_{1}, \ldots, i_{n-1}\right\}$ is a subset of $\{1, \ldots, n\}$ of cardinality $n-1$, which will be chosen depending on the type $j$ of the solution, $u_{i_{1}}, \ldots, u_{i_{n-1}}$ are integers and $I=O\left(\log ^{n-1} a\right)$.

Taking logarithms of the conjugates $h \in\{1, \ldots, n\} \backslash\{j\}$, we obtain a system of linear equations for the $u_{i_{k}} / I$ :

$$
\log \left|\beta^{(h)}\right|=\frac{u_{i_{1}}}{I} l_{i_{1}}^{(h)}+\ldots+\frac{u_{i_{n-1}}}{I} l_{i_{n-1}}^{(h)}, \quad h \neq j .
$$

Cramer's rule yields

$$
R \frac{u_{i_{k}}}{I}=\left|\begin{array}{ccccccc}
l_{i_{1}}^{(1)} & \ldots & l_{i_{k-1}}^{(1)} & \log \left|\beta^{(1)}\right| & l_{i_{k+1}}^{(1)} & \ldots & l_{i_{n-1}}^{(1)} \\
\vdots & \ddots & \vdots & \vdots & \vdots & \ddots & \vdots \\
l_{i_{1}}^{(n)} & \ldots & l_{i_{k-1}}^{(n)} & \log \left|\beta^{(n)}\right| & l_{i_{k+1}}^{(n)} & \ldots & l_{i_{n-1}}^{(n)}
\end{array}\right|
$$

where the $j$ th row is omitted and $R$ denotes the determinant of the system matrix, which is (up to sign) the regulator $R_{G}$ estimated in Lemma 9.

Applying (19) we obtain

$$
R \frac{u_{i_{k}}}{I}=M_{j, i_{k}} \log |y|+\Delta_{j, k} R+O\left(\frac{\log ^{n-2} a}{a^{e_{j}+d_{2}}}\right),
$$

where $\Delta_{j, k}= \pm 1$ if $j \notin\left\{i_{1}, \ldots, i_{k-1}, i_{k+1}, \ldots, i_{n-1}\right\}$ and 0 otherwise, and

$$
M_{j, i_{k}}=\left|\begin{array}{ccccccc}
l_{i_{1}}^{(1)} & \ldots & l_{i_{k-1}}^{(1)} & 1 & l_{i_{k+1}}^{(1)} & \ldots & l_{i_{n-1}}^{(1)} \\
\vdots & \ddots & \vdots & \vdots & \vdots & \ddots & \vdots \\
l_{i_{1}}^{(n)} & \ldots & l_{i_{k-1}}^{(n)} & 1 & l_{i_{k+1}}^{(n)} & \ldots & l_{i_{n-1}}^{(n)}
\end{array}\right|,
$$

where the $j$ th row is omitted. 
By Lemma 8 we see that

$$
M_{j, i_{k}}=G_{j, i_{k}} \log ^{n-2} a+O\left(\frac{\log ^{n-3} a}{a}\right)
$$

for some integers $G_{j, i_{k}}$.

We will choose a suitable $\mathbb{Z}$-linear combination $v_{j}$ of $u_{i_{k}}, k=1, \ldots, n-1$, and $I$ depending on the type $j$ of the solution such that (21) yields

$$
R \frac{v_{j}}{I}=M_{j} \log |y|+O\left(\frac{\log ^{n-2} a}{a^{e_{j}+d_{2}}}\right)
$$

with

$$
M_{j}=O\left(\frac{\log ^{n-3} a}{a}\right) .
$$

In the next sections we will prove that our choice of the linear combination $v_{j}$ implies

$$
M_{j}=\Omega\left(\frac{1}{a^{e_{j}+d_{2}-1}}\right) .
$$

If (24) holds, it is clear from (22) that for sufficiently large values of $a, v_{j}$ does not vanish, since we assume $|y| \geq 2$. If $R v_{j} / I>0$, then $R v_{j} / I \geq|R| / I$ because $v_{j}$ is an integer. Then by (22), (17), and (18) we obtain

$$
M_{j} \log |y| \geq \frac{|R|}{I}-O\left(\frac{\log ^{n-2} a}{a^{e_{j}+d_{2}}}\right)=\Omega(1)
$$

which implies $\log |y|=\Omega\left(a / \log ^{n-3} a\right)$ by (23). If $R v_{j} / I<0$, an analogous argument holds. Therefore, in order to prove Proposition 10 it suffices to show (23) and (24) for a suitable $v_{j}$.

6. Cases $3 \leq j \leq n$. We choose $\left(i_{1}, \ldots, i_{n-1}\right)=(1,3, \ldots, n)$ and $v_{j}:=u_{1}$. Our task is to prove (23) and (24) for

$$
M_{j}=\left|\begin{array}{cccc}
1 & l_{3}^{(1)} & \ldots & l_{n}^{(1)} \\
\vdots & \vdots & \ddots & \vdots \\
1 & l_{3}^{(n)} & \ldots & l_{n}^{(n)}
\end{array}\right|,
$$

where the $j$ th row is omitted. We note that we will not use the technical hypothesis (5) in this section.

We subtract the second row from the first and obtain

$$
M_{j}=\left|\begin{array}{cccc}
0 & l_{3}^{(1)}-l_{3}^{(2)} & \ldots & l_{n}^{(1)}-l_{n}^{(2)} \\
1 & l_{3}^{(2)} & \ldots & l_{n}^{(2)} \\
\vdots & \vdots & \ddots & \vdots \\
1 & l_{3}^{(n)} & \ldots & l_{n}^{(n)}
\end{array}\right|
$$


where the $j$ th row is omitted. For $3 \leq k \leq n$, we have

$$
\begin{aligned}
l_{k}^{(1)}-l_{k}^{(2)} & =\log \left|1-\left(1-\frac{\alpha^{(1)}-p_{k}}{\alpha^{(2)}-p_{k}}\right)\right|=\log \left(1+\frac{\alpha^{(2)}-\alpha^{(1)}}{p_{k}-\alpha^{(2)}}\right) \\
& =\frac{1}{a^{d_{k}-d_{2}}}\left(1+O\left(\frac{1}{a}\right)\right)
\end{aligned}
$$

hence $l_{k}^{(1)}-l_{k}^{(2)}=O(1 / a)$ and (23) is proved.

By Lemma 8, we obtain

$$
M_{j}=Q_{j}(\log a, 1 / a)+O\left(\frac{\log ^{n-3} a}{a^{e_{1}+d_{2}}}\right),
$$

where $Q_{j}(\log a, 1 / a)$ is some polynomial in $\log a$ and $1 / a$ with rational coefficients depending on the coefficients of the polynomials $p_{i}$.

We consider the coefficient of $\left(\log ^{n-3} a\right) / a^{d_{j}-d_{2}}$ in $Q_{j}$. We expand the determinant in (25) according to the first row and get

$$
M_{j}=\sum_{k=3}^{n}(-1)^{k}\left(l_{k}^{(1)}-l_{k}^{(2)}\right) N_{j k}=\sum_{k=3}^{n} \frac{(-1)^{k}}{a^{d_{k}-d_{2}}}\left(1+O\left(\frac{1}{a}\right)\right) N_{j k},
$$

where $N_{j k}$ are the corresponding minors. By (26) and (4), there is no contribution of the summands with $k>j$. For $k<j$, we have $\frac{N_{j k}}{\log ^{n-3} a}$

$$
=\left|\begin{array}{ccccccccccc}
1 & d_{3} & \ldots & d_{k-1} & d_{k+1} & \ldots & d_{j-1} & d_{j} & d_{j+1} & \ldots & d_{n} \\
1 & -e_{3} & \ldots & d_{k-1} & d_{k+1} & \ldots & d_{j-1} & d_{j} & d_{j+1} & \ldots & d_{n} \\
\vdots & \vdots & \ddots & \vdots & \vdots & \ddots & \vdots & \vdots & \vdots & \ddots & \vdots \\
1 & d_{k-1} & \ldots & -e_{k-1} & d_{k+1} & \ldots & d_{j-1} & d_{j} & d_{j+1} & \ldots & d_{n} \\
1 & d_{k} & \ldots & d_{k} & d_{k+1} & \ldots & d_{j-1} & d_{j} & d_{j+1} & \ldots & d_{n} \\
1 & d_{k+1} & \ldots & d_{k+1} & -e_{k+1} & \ldots & d_{j-1} & d_{j} & d_{j+1} & \ldots & d_{n} \\
\vdots & \vdots & \ddots & \vdots & \vdots & \ddots & \vdots & \vdots & \vdots & \ddots & \vdots \\
1 & d_{j-1} & \ldots & d_{j-1} & d_{j-1} & \ldots & -e_{j-1} & d_{j} & d_{j+1} & \ldots & d_{n} \\
1 & d_{j+1} & \ldots & d_{j+1} & d_{j+1} & \ldots & d_{j+1} & d_{j+1} & -e_{j+1} & \ldots & d_{n} \\
\vdots & \vdots & \ddots & \vdots & \vdots & \ddots & \vdots & \vdots & \vdots & \ddots & \vdots \\
1 & d_{n} & \ldots & d_{n} & d_{n} & \ldots & d_{n} & d_{n} & d_{n} & \ldots & -e_{n}
\end{array}\right|
$$

Subtracting $d_{l}$ times the first column from the column corresponding to $l$, 
we obtain, with $h_{l}=-e_{l}-d_{l}(3 \leq l \leq n)$,

$$
N_{j k}=\log ^{n-3} a\left|\begin{array}{ccccccccccc}
1 & 0 & \ldots & 0 & 0 & \ldots & 0 & 0 & 0 & \ldots & 0 \\
1 & h_{3} & \ldots & 0 & 0 & \ldots & 0 & 0 & 0 & \ldots & 0 \\
\vdots & \vdots & \ddots & \vdots & \vdots & \ddots & \vdots & \vdots & \vdots & \ddots & \vdots \\
1 & * & \ldots & h_{k-1} & 0 & \ldots & 0 & 0 & 0 & \ldots & 0 \\
1 & * & \ldots & * & 0 & \ldots & 0 & 0 & 0 & \ldots & 0 \\
1 & * & \ldots & * & h_{k+1} & \ldots & 0 & 0 & 0 & \ldots & 0 \\
\vdots & \vdots & \ddots & \vdots & \vdots & \ddots & \vdots & \vdots & \vdots & \ddots & \vdots \\
1 & * & \ldots & * & * & \ldots & h_{j-1} & 0 & 0 & \ldots & 0 \\
1 & * & \ldots & * & * & \ldots & * & * & h_{j+1} & \ldots & 0 \\
\vdots & \vdots & \ddots & \vdots & \vdots & \ddots & \vdots & \vdots & \vdots & \ddots & \vdots \\
1 & * & \ldots & * & * & \ldots & * & * & * & \ldots & h_{n}
\end{array}\right|
$$

where $*$ stands for constants which are not important to us. Hence for $k<j$, $N_{j k}=O\left(\left(\log ^{n-4} a\right) / a\right)$, and there is no contribution to the term considered.

In the same fashion we get, for $k=j$,

$$
\begin{aligned}
& N_{j j}=\log ^{n-3} a\left|\begin{array}{ccccccc}
1 & 0 & \ldots & 0 & 0 & \ldots & 0 \\
1 & h_{3} & \ldots & 0 & 0 & \ldots & 0 \\
\vdots & \vdots & \ddots & \vdots & \vdots & \ddots & \vdots \\
1 & * & \ldots & h_{j-1} & 0 & \ldots & 0 \\
1 & * & \ldots & * & h_{j+1} & \ldots & 0 \\
\vdots & \vdots & \ddots & \vdots & \vdots & \ddots & \vdots \\
1 & * & \ldots & * & * & \ldots & h_{n}
\end{array}\right|+O\left(\frac{\log ^{n-4} a}{a}\right) \\
&=\log ^{n-3} a \prod_{\substack{l=3 \\
l \neq j}}^{n}\left(-e_{l}-d_{l}\right)+O\left(\frac{\log ^{n-4} a}{a}\right) .
\end{aligned}
$$

Thus the coefficient of $\left(\log ^{n-3} a\right) / a^{d_{j}-d_{2}}$ in (27) does not vanish, which implies (24) since $d_{j}-d_{2}<e_{1}+d_{2}$ by (4).

7. Cases $j=1$ and $j=2$. In both cases, we choose $\left(i_{1}, \ldots, i_{n-1}\right)=$ $(1,2,4, \ldots, n)$. We set

$$
j^{\prime}:= \begin{cases}2 & \text { if } j=1 \\ 1 & \text { if } j=2\end{cases}
$$

and get 


$$
\begin{gathered}
M_{j, j}=(-1)^{j^{\prime}}\left|\begin{array}{ccccc}
1 & l_{j^{\prime}}^{\left(j^{\prime}\right)} & l_{4}^{\left(j^{\prime}\right)} & \ldots & l_{n}^{\left(j^{\prime}\right)} \\
1 & l_{j^{\prime}}^{(3)} & l_{4}^{(3)} & \ldots & l_{n}^{(3)} \\
\vdots & \vdots & \vdots & \ddots & \vdots \\
1 & l_{j^{\prime}}^{(n)} & l_{4}^{(n)} & \ldots & l_{n}^{(n)}
\end{array}\right|, \\
M_{j, j^{\prime}}=(-1)^{j}\left|\begin{array}{ccccc}
1 & l_{j}^{\left(j^{\prime}\right)} & l_{4}^{\left(j^{\prime}\right)} & \ldots & l_{n}^{\left(j^{\prime}\right)} \\
1 & l_{j}^{(3)} & l_{4}^{(3)} & \ldots & l_{n}^{(3)} \\
\vdots & \vdots & \vdots & \ddots & \vdots \\
1 & l_{j}^{(n)} & l_{4}^{(n)} & \ldots & l_{n}^{(n)}
\end{array}\right|
\end{gathered}
$$

and $\Delta_{j, j}=1$ and $\Delta_{j, j^{\prime}}=0$. We now choose the linear combination $v_{j}:=$ $\left(d_{2}-d_{3}\right)\left(u_{j}-I\right)+\left(d_{3}+e_{1}\right) u_{j^{\prime}}$, which yields

$$
M_{j}=\left|\begin{array}{ccccc}
1 & \left(d_{2}-d_{3}\right) l_{j^{\prime}}^{\left(j^{\prime}\right)}+\left(d_{3}+e_{1}\right) l_{j}^{\left(j^{\prime}\right)} & l_{4}^{\left(j^{\prime}\right)} & \ldots & l_{n}^{\left(j^{\prime}\right)} \\
1 & \left(d_{2}-d_{3}\right) l_{j^{\prime}}^{(3)}+\left(d_{3}+e_{1}\right) l_{j}^{(3)} & l_{4}^{(3)} & \ldots & l_{n}^{(3)} \\
\vdots & \vdots & \vdots & \ddots & \vdots \\
1 & \left(d_{2}-d_{3}\right) l_{j^{\prime}}^{(n)}+\left(d_{3}+e_{1}\right) l_{j}^{(n)} & l_{4}^{(n)} & \ldots & l_{n}^{(n)}
\end{array}\right| .
$$

By subtracting appropriate multiples of column 1 from the other columns such that the first row becomes $\left(\begin{array}{llll}1 & 0 & \ldots & 0\end{array}\right)$ and by expanding the determinant according to the first row, we obtain

$$
M_{j}=\left|\begin{array}{cccc}
\nu_{3} & l_{4}^{(3)}-l_{4}^{\left(j^{\prime}\right)} & \ldots & l_{n}^{(3)}-l_{n}^{\left(j^{\prime}\right)} \\
\vdots & \vdots & \ddots & \vdots \\
\nu_{n} & l_{4}^{(n)}-l_{4}^{\left(j^{\prime}\right)} & \ldots & l_{n}^{(n)}-l_{n}^{\left(j^{\prime}\right)}
\end{array}\right|,
$$

where $\nu_{i}:=\left(d_{2}-d_{3}\right)\left(l_{j^{\prime}}^{(i)}-l_{j^{\prime}}^{\left(j^{\prime}\right)}\right)+\left(d_{3}+e_{1}\right)\left(l_{j}^{(i)}-l_{j}^{\left(j^{\prime}\right)}\right), i=3, \ldots, n$.

By Lemma 8 we see that

$$
\begin{aligned}
\nu_{3} & =\left(d_{2}-d_{3}\right)\left(l_{j^{\prime}}^{(3)}-l_{j^{\prime}}^{\left(j^{\prime}\right)}\right)+\left(d_{3}+e_{1}\right)\left(l_{j}^{(3)}-l_{j}^{\left(j^{\prime}\right)}\right) \\
& =\left(d_{2}-d_{3}\right)\left(d_{3}+e_{1}\right) \log a+\left(d_{3}+e_{1}\right)\left(d_{3}-d_{2}\right) \log a+O\left(a^{-1}\right)=O\left(a^{-1}\right)
\end{aligned}
$$

and

$$
l_{k}^{(3)}-l_{k}^{\left(j^{\prime}\right)}=d_{k} \log a-d_{k} \log a+O\left(a^{-1}\right)=O\left(a^{-1}\right), \quad 4 \leq k \leq n,
$$

which proves (23).

In order to prove (24) we note that it is sufficient to show that the coefficient $C_{j}$ of $\log ^{n-3} a$ in the expansion of $M_{j}$ according to Lemma 8 satisfies $C_{j}=\Omega\left(a^{-e_{1}-d_{2}+1}\right)$. 
Lemma 11. Assume $C_{j}=O\left(a^{-e_{1}-d_{2}}\right)$. Then for $3 \leq k \leq n$ we have

$$
\begin{aligned}
C_{j}= & \prod_{l=4}^{k}\left(-e_{l}-d_{l}\right) \\
& \times\left|\begin{array}{ccccc}
g_{k, k, j} & g_{k+1, k, j} & g_{k+2, k, j} & \ldots & g_{n, k, j} \\
\mu_{k+1, k} & -e_{k+1}-d_{k+1} & 0 & \ldots & 0 \\
\mu_{k+2, k} & d_{k+2}-d_{k+1} & -e_{k+2}-d_{k+2} & \ldots & 0 \\
\vdots & \vdots & \vdots & \ddots & \vdots \\
\mu_{n, k} & d_{n}-d_{k+1} & d_{n}-d_{k+2} & \ldots & -e_{n}-d_{n}
\end{array}\right|,
\end{aligned}
$$

where

$$
\begin{gathered}
g_{h, k, j}=l_{h}^{(3)}-l_{h}^{\left(j^{\prime}\right)}, \quad k+1 \leq h \leq n, \\
g_{k, k, j} \text { is a } \mathbb{Z} \text {-linear combination of } l_{i}^{(m)}, \quad m \in\left\{j^{\prime}, 3\right\}, i \neq m, \\
g_{k, k, j}=O(1 / a), \\
\mu_{h, k} \in \mathbb{N}, \quad k+1 \leq h \leq n .
\end{gathered}
$$

Proof. We observe that $(29 \mathrm{c})$ and Lemma 8 imply that there are nonnegative integers $\lambda_{i, m, k, j}^{+}$and $\lambda_{i, m, k, j}^{-}$such that

$$
g_{k, k, j}=\sum_{(m, i)}\left(\lambda_{i, m, k, j}^{+}-\lambda_{i, m, k, j}^{-}\right) l_{i}^{(m)}=\widetilde{g}_{k, k, j}+O\left(\frac{1}{a^{e_{1}+d_{2}}}\right),
$$

where

$$
\widetilde{g}_{k, k, j}=\log \left|1+\frac{\prod_{(m, i)}\left(p_{i}-p_{m}\right)^{\lambda_{i, m, k, j}^{+}-} \prod_{(m, i)}\left(p_{i}-p_{m}\right)^{\lambda_{i, m, k, j}^{-}}}{\prod_{(m, i)}\left(p_{i}-p_{m}\right)^{\lambda_{i, m, k, j}^{-}}}\right|
$$

and where the indices $(i, m)$ range over $m \in\left\{j^{\prime}, 3\right\}, i \neq m$. (29d) asserts that the degree of the numerator is less than the degree of the denominator, hence there is a nonzero integer $\chi_{k, j}$ and an $s_{k, j} \in \mathbb{N}$ such that

$$
g_{k, k, j}=\frac{\chi_{k, j}}{a^{s_{k, j}}}\left(1+O\left(\frac{1}{a}\right)\right)+O\left(\frac{1}{a^{e_{1}+d_{2}}}\right) .
$$

We now prove the lemma by induction on $k$.

Let $k=3$. The definition of $C_{j}$, Lemma 8 and the fact that the first row in the determinant in (28) is $O\left(a^{-1}\right)$ necessitate a term containing $\log a$ in rows 2 to $n-2$ in order to get $\log ^{n-3} a$. Since $l_{h}^{(k)}-l_{h}^{\left(j^{\prime}\right)}=O\left(a^{-1}\right)$ for $h>k$, we obtain the representation (29a). (29b) is clear and

$$
g_{3,3, j}=\left(d_{2}-d_{3}\right)\left(l_{j^{\prime}}^{(3)}+\sum_{h \neq j^{\prime}} l_{h}^{\left(j^{\prime}\right)}\right)+\left(d_{3}+e_{1}\right)\left(l_{j}^{(3)}-l_{j}^{\left(j^{\prime}\right)}\right)
$$

implies (29c). (29d) has already been observed and (29e) follows from (4) 
since for $4 \leq h \leq n$,

$$
\begin{aligned}
\mu_{h, 3} & =\left(d_{2}-d_{3}\right)\left(d_{h}+e_{1}\right)+\left(d_{3}+e_{1}\right)\left(d_{h}-d_{2}\right) \\
& =\left(d_{2}+e_{1}\right)\left(d_{h}-d_{3}\right) \in \mathbb{N} .
\end{aligned}
$$

Assume now that the lemma holds for some $3 \leq k \leq n-1$. Then by (29a), (30) and Lemma 8 we have, with $s_{k, j}^{\prime}:=\min \left\{d_{k+1}-d_{3}, s_{k, j}\right\}+1$,

$$
\begin{aligned}
& C_{j}=\prod_{h=4}^{k}\left(-e_{h}-d_{h}\right) \\
& \times\left|\begin{array}{ccccc}
\frac{\chi_{k, j}}{a^{s_{k, j}}} & -\frac{1}{a^{d_{k+1}-d_{3}}} & 0 & \ldots & 0 \\
\mu_{k+1, k} & -e_{k+1}-d_{k+1} & 0 & \ldots & 0 \\
\mu_{k+2, k} & d_{k+2}-d_{k+1} & -e_{k+2}-d_{k+2} & \ldots & 0 \\
\vdots & \vdots & \vdots & \ddots & \vdots \\
\mu_{n, k} & d_{n}-d_{k+1} & d_{n}-d_{k+2} & \ldots & -e_{n}-d_{n}
\end{array}\right| \\
& +O\left(\frac{1}{a^{s_{k, j}^{\prime}}}\right) \\
& =\prod_{h=4}^{k}\left(-e_{h}-d_{h}\right)\left(\frac{\chi_{k, j}}{a^{s_{k, j}}}\left(-e_{k+1}-d_{k+1}\right)+\frac{1}{a^{d_{k+1}-d_{3}}} \mu_{k+1, k}\right) \\
& \times \prod_{h=k+2}^{n}\left(-e_{h}-d_{h}\right)+O\left(\frac{1}{a^{s_{k, j}^{\prime}}}\right) .
\end{aligned}
$$

By $(29 \mathrm{e}),(4)$ and $\chi_{k, j} \neq 0$, we see that if $s_{k, j} \neq d_{k+1}-d_{3}$, then (24) follows immediately. So we only have to consider the case

$$
s_{k, j}=d_{k+1}-d_{3}=: s_{k}, \quad \chi_{k, j}=\frac{\mu_{k+1, k}}{e_{k+1}+d_{k+1}}=: \chi_{k} \in \mathbb{N} .
$$

(29a) yields

$$
\begin{aligned}
& C_{j}=\prod_{h=4}^{k}\left(-e_{h}-d_{h}\right) \\
& \times\left|\begin{array}{ccccc}
g_{k, k, j}+\chi_{k} g_{k+1, k, j} & g_{k+1, k, j} & g_{k+2, k, j} & \ldots & g_{n, k, j} \\
\mu_{k+1, k}-\chi_{k}\left(e_{k+1}+d_{k+1}\right) & -e_{k+1}-d_{k+1} & 0 & \ldots & 0 \\
\mu_{k+2, k}+\chi_{k}\left(d_{k+2}-d_{k+1}\right) & d_{k+2}-d_{k+1} & -e_{k+2}-d_{k+2} & \ldots & 0 \\
\vdots & \vdots & \vdots & \ddots & \vdots \\
\mu_{n, k}+\chi_{k}\left(d_{n}-d_{k+1}\right) & d_{n}-d_{k+1} & d_{n}-d_{k+2} & \ldots & -e_{n}-d_{n}
\end{array}\right|
\end{aligned}
$$

and by (33) we obtain 


$$
C_{j}=\prod_{h=4}^{k}\left(-e_{h}-d_{h}\right)
$$

$$
\times\left|\begin{array}{ccccc}
g_{k+1, k+1, j} & g_{k+1, k, j} & g_{k+2, k+1, j} & \ldots & g_{n, k+1, j} \\
0 & -e_{k+1}-d_{k+1} & 0 & \ldots & 0 \\
\mu_{k+2, k+1} & d_{k+2}-d_{k+1} & -e_{k+2}-d_{k+2} & \ldots & 0 \\
\vdots & \vdots & \vdots & \ddots & \vdots \\
\mu_{n, k+1} & d_{n}-d_{k+1} & d_{n}-d_{k+2} & \ldots & -e_{n}-d_{n}
\end{array}\right|
$$

where

$$
\begin{array}{rlrl}
g_{h, k+1, j} & :=g_{h, k, j}, & & k+2 \leq h \leq n, \\
g_{k+1, k+1, j} & :=g_{k, k, j}+\chi_{k} g_{k+1, k, j}, & \\
\mu_{h, k+1}:=\mu_{h, k}+\chi_{k}\left(d_{h}-d_{k+1}\right), & k+2 \leq h \leq n,
\end{array}
$$

hence we have

$$
C_{j}=\prod_{h=4}^{k+1}\left(-e_{h}-d_{h}\right)\left|\begin{array}{cccc}
g_{k+1, k+1, j} & g_{k+2, k+1, j} & \ldots & g_{n, k+1, j} \\
\mu_{k+2, k+1} & -e_{k+2}-d_{k+2} & \ldots & 0 \\
\vdots & \vdots & \ddots & \vdots \\
\mu_{n, k+1} & d_{n}-d_{k+2} & \ldots & -e_{n}-d_{n}
\end{array}\right|
$$

which implies (29a) for $k+1$. (34a) yields (29b), (34b) and (29b) result in $(29 \mathrm{c})$ and $(29 \mathrm{~d})$, and $(34 \mathrm{c}),(33)$, and (4) give $(29 \mathrm{e})$ for $k+1$.

By $(34 \mathrm{c})$ and (33) we see that the recursive definition of $\psi_{i}$ in (3) matches the definition of $\chi_{i}$, so that $\chi_{i}=\psi_{i}$ for $3 \leq i \leq n-1$.

(34b) and (31) yield

$$
\begin{aligned}
g_{n, n, j}= & {\left[\left(d_{3}+e_{1}\right) l_{j}^{(3)}+\sum_{k=4}^{n} \psi_{k-1} l_{k}^{(3)}\right] } \\
& -\left[\left(e_{1}+2 d_{3}-d_{2}\right) l_{j}^{\left(j^{\prime}\right)}+\left(d_{3}-d_{2}\right) l_{3}^{\left(j^{\prime}\right)}+\left(d_{3}-d_{2}\right) l_{j^{\prime}}^{(3)}\right. \\
& \left.+\sum_{k=4}^{n}\left(\psi_{k-1}+d_{3}-d_{2}\right) l_{k}^{\left(j^{\prime}\right)}\right] .
\end{aligned}
$$

From Lemma 8 follows

$$
g_{n, n, j}+O\left(\frac{1}{a^{e_{1}+d_{2}}}\right)=\log \left(\frac{Q_{j}^{+}}{Q_{j}^{-}}\right)=\log \left(1+\frac{Q_{j}^{+}-Q_{j}^{-}}{Q_{j}^{-}}\right),
$$

where $Q_{j}^{+}$and $Q_{j}^{-}$have been defined in Theorems 1 and 2. Assume that (24) does not hold. This implies $C_{j}=O\left(a^{-e_{1}-d_{2}}\right)$, and so we can apply Lemma 11 to deduce that $\psi_{i} \in \mathbb{N}, 3 \leq i \leq n-1$, and $g_{n, n, j}=O\left(a^{-e_{1}-d_{2}}\right)$. 
But in this case, (5) can be used to show $g_{n, n, j}=\Omega\left(a^{-e_{1}-d_{2}+1}\right)$, which yields a contradiction. Hence (24) and therefore Proposition 10 are proved.

8. Large solutions. To exclude "large" solutions linear forms in logarithms can be employed in the usual procedure. Since we do not care about constants, we will use the explicit bound for the solutions of Thue equations due to Bugeaud and Györy [5].

Theorem 12 (Bugeaud-Györy [5]). Let $F \in \mathbb{Z}[X, Y]$ be a homogeneous irreducible polynomial of degree $n \geq 3$ and $0 \neq m \in \mathbb{Z}$. Let $B \geq \max \{|m|, e\}$, $\alpha$ be a zero of $F(X, 1), K:=\mathbb{Q}(\alpha), R:=R_{K}$ the regulator and $r$ the unit rank of $K$. Let $H \geq 3$ be an upper bound for the absolute values of the coefficients of $F$. Then all solutions $(x, y) \in \mathbb{Z}^{2}$ of

$$
F(x, y)=m
$$

satisfy

$$
\max \{|x|,|y|\}<\exp \left(C_{1} \cdot R \cdot \max \{\log R, 1\} \cdot(R+\log (H B))\right),
$$

where

$$
C_{1}=C_{1}(n, r)=3^{r+27}(r+1)^{7 r+19} n^{2 n+6 r+14} .
$$

In our situation, we have $m=1, B=e, R_{K} \leq R_{\mathfrak{D}} \leq R_{G}=O\left(\log ^{n-1} a\right)$ by (17), $r=n-1, H=a^{O(1)}$, which yields

$$
\log |y|=O\left(\log ^{2 n-1} a\right) .
$$

This is a contradiction to Proposition 10, hence there are no solutions with $|y| \geq 2$ for sufficiently large values of $a$, which proves Theorems 1 and 2 .

9. Proof of Corollary 3. If $\delta_{4}=1$, we have $0<\psi_{3}=\left(e_{2}+d_{2}\right) /\left(e_{4}+d_{4}\right)$ $<1$ so that $\psi_{3} \notin \mathbb{N}$ and we are done. Therefore, we can assume $d_{4}-d_{3}>1$. Calculating $Q_{j}^{+}$and $Q_{j}^{-}$, we obtain

$$
\begin{array}{r}
Q_{j}^{+}=a^{\operatorname{deg} Q_{j}^{+}}\left(1+\left(\left(e_{1}+d_{3}\right)\left(c_{3}-\delta_{j 2} \delta_{3}\right)+\sum_{k=4}^{n} \psi_{k-1} c_{k}\right) \frac{1}{a}+O\left(\frac{1}{a^{2}}\right)\right), \\
Q_{j}^{-}=a^{\operatorname{deg} Q_{j}^{-}}\left(1+\left(\left(e_{1}-d_{2}+2 d_{3}\right)\left(c_{2}-\delta_{2}\right)+2\left(d_{3}-d_{2}\right)\left(c_{3}-\delta_{j 1} \delta_{3}\right)\right.\right. \\
\left.\left.+\sum_{k=4}^{n}\left(\psi_{k-1}+d_{3}-d_{2}\right) c_{k}\right) \frac{1}{a}+O\left(\frac{1}{a^{2}}\right)\right),
\end{array}
$$

where $\operatorname{deg} Q_{j}^{+}=\operatorname{deg} Q_{j}^{-}=d_{3}\left(e_{1}+d_{3}\right)+\sum_{k=4}^{n} d_{k} \psi_{k-1}, \delta_{i}$ has been defined in Corollary 3 and $\delta_{k l}$ denotes the usual Kronecker delta, $\delta_{k l}=1$ if $k=l$ and 0 otherwise. 
We easily see that (11) implies $\operatorname{deg}\left(Q_{j}^{+}-Q_{j}^{-}\right)=\operatorname{deg} Q_{j}^{-}-1>\operatorname{deg} Q_{j}^{-}-$ $e_{1}-d_{2}$, hence Theorem 1 (or Theorem 2) can be applied.

\section{References}

[1] A. Baker, Contribution to the theory of Diophantine equations. I. On the representation of integers by binary forms, Philos. Trans. Roy. Soc. London Ser. A 263 (1968), 173-191.

[2] C. Batut, K. Belabas, D. Benardi, H. Cohen and M. Olivier, User's Guide to PARI$G P$, available at ftp://megrez.math.u-bordeaux.fr/pub/pari/manuals/users.ps.gz, 2000.

[3] M. A. Bennett, Rational approximation to algebraic numbers of small height: The Diophantine equation $\left|a x^{n}-b y^{n}\right|=1$, J. Reine Angew. Math., to appear.

[4] Yu. Bilu and G. Hanrot, Solving Thue equations of high degree, J. Number Theory 60 (1996), 373-392.

[5] Y. Bugeaud and K. Györy, Bounds for the solutions of Thue-Mahler equations and norm form equations, Acta Arith. 74 (1996), 273-292.

[6] F. Halter-Koch, G. Lettl, A. Pethő and R. F. Tichy, Thue equations associated with Ankeny-Brauer-Chowla number fields, J. London Math. Soc. (2) 60 (1999), 1-20.

[7] C. Heuberger, On families of parametrized Thue equations, J. Number Theory 76 (1999), 45-61.

[8] C. Heuberger, A. Pethö and R. F. Tichy, Complete solution of parametrized Thue equations, Acta Math. Inform. Univ. Ostraviensis 6 (1998), 93-113.

[9] C. Heuberger and R. F. Tichy, Effective solution of families of Thue equations containing several parameters, Acta Arith. 91 (1999), 147-163.

[10] S. Lang, Elliptic Curves: Diophantine Analysis, Grundlehren Math. Wiss. 23, Springer, Berlin, 1978.

[11] E. Lee, Studies on Diophantine equations, Ph.D. thesis, Cambridge Univ., 1992.

[12] M. Mignotte, Pethő's cubics, Publ. Math. Debrecen 56 (2000), 481-505.

[13] M. Mignotte and N. Tzanakis, On a family of cubics, J. Number Theory 39 (1991), 41-49.

[14] A. Pethő und R. Schulenberg, Effektives Lösen von Thue Gleichungen, Publ. Math. Debrecen 34 (1987), 189-196.

[15] M. Pohst and H. Zassenhaus, Algorithmic Algebraic Number Theory, Cambridge Univ. Press, Cambridge, 1989.

[16] R. Remak, Über die Abschätzung des absoluten Betrages des Regulators eines algebraischen Zahlkörpers nach unten, J. Reine Angew. Math. 167 (1932), 360-378.

[17] E. Thomas, Complete solutions to a family of cubic Diophantine equations, J. Number Theory 34 (1990), 235-250.

[18] —, Solutions to certain families of Thue equations, ibid. 43 (1993), 319-369.

[19] A. Thue, Über Annäherungswerte algebraischer Zahlen, J. Reine Angew. Math. 135 (1909), 284-305.

[20] N. Tzanakis and B. M. M. de Weger, On the practical solution of the Thue equation, J. Number Theory 31 (1989), 99-132. 
[21] W. Windsteiger, Using GRÖBNER as a "black box", Technical Report 71, RISCLinz, University of Linz, 1993. Available at http://www.risc.uni-linz.ac.at/people/ wwindste/Public/Reports/GBBB/master.ps.gz.

Institut für Mathematik B

Technische Universität Graz

Steyrergasse 30/II

A-8010 Graz, Austria

E-mail: clemens.heuberger@tugraz.at

http://finanz.math.tu-graz.ac.at/ ${ }^{\sim}$ cheub/

Received on 18.4.2000

and in revised form on 3.8.2000 